\title{
Squeeze flow magnetorheology
}

\author{
Juan de Vicente, ${ }^{\text {a) }}$ José Antonio Ruiz-López, Efrén Andablo-Reyes, \\ Juan Pablo Segovia-Gutiérrez, and Roque Hidalgo-Alvarez \\ Department of Applied Physics, Faculty of Sciences, University of Granada, \\ C/Fuentenueva s/n, 18071 Granada, Spain
}

(Received 28 October 2010; final revision received 15 March 2011;

published 13 April 2011)

\begin{abstract}
Synopsis
This paper is concerned with an investigation of the rheological performance of magnetorheological fluids under squeeze flow. Preliminary results on Newtonian fluids are first compared to Stefan's equation. Then, unidirectional monotonic compression tests are carried out in the presence of uniaxial external magnetic fields at slow compression rates under constant volume operation. Results are compared to Bingham plastic, biviscous, and single chain micromechanical squeeze flow models. Measurements using combined deformation modes (compression+small-strain oscillatory shear) suggest a compression-induced shear strengthen effect up to strains of $\sim 0.5$. Particle-level dynamic simulations are in qualitatively good agreement with experimental observations. (C) 2011 The Society of Rheology. [DOI: 10.1122/1.3574932]
\end{abstract}

\section{INTRODUCTION}

Magnetorheological (MR) and electrorheological (ER) fluids are field-responsive colloids that show a rapid increase in viscosity upon the application of an external magnetic or electric field, respectively [Ginder (1998); Rankin et al. (1998); Bossis et al. (2002); Park et al. (2010); de Vicente et al. (2011)]. The reason for this is the formation of particle clusters aligned in the field direction. As a starting point, their flow behavior is typically modeled by the Bingham-fluid-like equation where, in the case of steady shear flow, the stress is given by

$$
\tau=\tau_{y}+\eta_{p} \dot{\gamma}
$$

Here, $\tau_{y}$ is the yield shear stress, $\eta_{p}$ is the plastic viscosity, and $\dot{\gamma}$ is the shear rate. In this work we will focus on MR fluids. However, many of the results presented below can be easily extended to ER fluids.

Currently, an important gap exists between commercial requirements for the strength of field-responsive fluids and their performance levels. While significant work has been done to enhance the fluid formulation [Bossis et al. (2002); de Vicente et al. (2003); Vereda et al. (2007, 2009); de Vicente et al. (2010)], much less efforts have been made to improve the design of devices, most of them being restricted to shearing operation regimes [Olabi and Grunwald (2007)]. Most devices that use MR fluids can be classified as

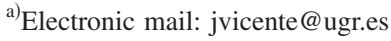


(a) Pressure-driven

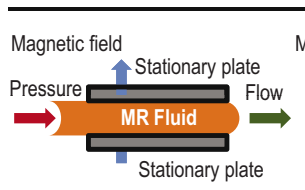

(b) Direct-shear

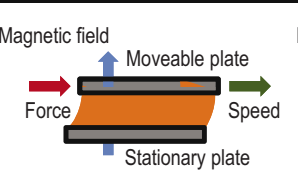

(c) Squeeze

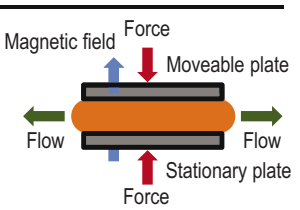

FIG. 1. Basic operational modes for controllable MR fluid devices. Adapted from A. G. Olabi and A. Grunwald, Mater. Des. 28, 2658 (2007). Copyright (C2007, with permission from Elsevier.

having fixed poles (pressure-driven flow mode), relatively movable poles (direct-shear and squeeze-film flow modes), or a combination of these modes [Jolly and Carlson (1996)]. Diagrams of these three basic operational standard flow modes are shown in Fig. 1. Interestingly, it has been documented that field-responsive fluids show yield stresses in the order of direct-shear $<$ pressure-driven $<$ squeeze flow mode [Havelka and Pialet (1996)].

Classical direct-shear mode has been studied thoroughly in the literature under dynamic [de Gans et al. (1999); de Vicente et al. (2005); Saldivar-Guerrero et al. (2006); Ekwebelam and See (2007); de Vicente and Ramírez (2007); de Vicente et al. (2009, 2010); Ramos et al. (2010)] and steady shear [Volkova et al. (2000); de Vicente et al. (2004); Park et al. (2006); Bell et al. (2008); de Vicente et al. (2010); Ramos et al. (2011)] regimes, and several products are already present in the market [Olabi and Grunwald (2007)]. Pressure-driven flow mode investigations have also been reported because much larger and realistic shear rates can be imposed (up to $20000 \mathrm{~s}^{-1}$, in contrast to conventional rotational rheometry that encompasses shear rates only up to a few thousand 1/s) [Wang and Gordaninejad (2006)]. Surprisingly, relatively limited attention has been given to the behavior of MR fluids in squeeze flow mode, even though it has been suggested that the yield stress that could be achieved would be ten times larger than that attainable with either the direct-shear or pressure-driven flow modes [Zhang et al. (2004)]. Furthermore, a compression-assisted-aggregation process has been demonstrated to enhance the yield shear stress in field-responsive fluids by the formation of thick columns with strong and robust ends (MR fluids [Tang et al. (2000)] and ER fluids [Tao et al. (2002)]). Despite their importance, a complete understanding of the mechanical properties of MR fluids under valve or squeeze flow behavior is still missing in the literature [McIntyre and Filisko (2010)].

In this paper the squeeze flow (i.e., biaxial elongational flow) behavior of MR fluids is studied. First, the experimental procedure for investigating the normal force was checked by measuring forces with model Newtonian fluids. Then, we focused on the squeeze flow behavior of MR fluids in low Peclet numbers (slow plate motion) where "filtration" is expected to be significant [McIntyre and Filisko (2010)]. Although the interparticle interaction is the key issue in the field-responsive effect-especially at small compressive strains - the structure of particle aggregates is known to be also important in the MR mechanism. In this sense we also investigated the structure evolution under compression through combined small-amplitude oscillatory shear (SAOS) and squeeze flows as well as using particle dynamics simulations. The paper is structured as follows: In Sec. II we review the state-of-the-art of squeeze flow behavior of field-responsive fluids. In Sec. III we illustrate some of the most relevant squeeze flow theories for Newtonian and plastic fluids. In Sec. IV we describe the experiments, and in Sec. V we show the details of the particle-level MR simulations. Results are presented Sec. VI. We end with some conclusions in Sec. VII. 


\section{BACKGROUND}

A seminal paper published by Stanway et al. (1987) is probably the first to demonstrate the feasibility of employing field-responsive (ER) fluids under squeeze flow. Here, tensile and compressive forces were found to be considerably larger than those developed in shearing flows. This was later confirmed through experiments by Monkman (1995) and Gong and Lim (1996). A short time later, vibration control applications followed by the construction and testing of ER squeeze-based damping devices [Stanway et al. (1992)] and engine mounts [Sproston et al. (1994a)] were found. On the theoretical side both macroscopic and microscopic approaches have been used. A biviscous model, inspired in earlier work on plastic fluids by Gartling and Phan-Thien (1984), was successfully used to explain the dynamic performance of ER fluids in squeeze [Williams et al. (1993); Sproston et al. (1994a, 1994b)] and unidirectional monotonic compression for large gap separations [Tian et al. (2003); Meng and Filisko (2005)]. Some years later, inertia was included in the theoretical derivation [Lee and Wen (2002)], and an iterative approach for the yield stress was incorporated to the model rather than using the classical power law expression [El Wahed et al. (2003)]. Lukkarinen and Kaski $(1996,1998)$ employed computer simulation methods in a comparative study of the mechanical properties of model ER structures under various dynamical loading conditions (shear, compression, and tension). It was demonstrated that compressive loading transferred the largest force. Unlike simulations under shear flow, in squeeze flow simulations it is not easy to apply periodic boundary conditions, so in general the number of particles needs to be increased. To avoid this problem a message passing interface parallel processing technique was employed by Kim et al. (1999).

An investigation on the application of ER fluids to control vibration under conditions of constant voltage and constant electric field was reported by El Wahed et al. (1998). The effects of electric field strength, particle concentration, and continuous phase viscosity were investigated by Chu et al. (2000). Special emphasis was paid to the appearance of normal stress fluctuations due to the deformation of field-induced chainlike structures under the field. The effect of an ac electric field, with a tunable waveform, on the performance of an ER fluid in oscillatory squeeze flow was investigated by El Wahed et al. (2000). The biviscous model was found to be equally valid for the ac case and for the dc case reported by El Wahed et al. (1998). See et al. (1999) measured field-induced force waveforms in ER fluids under oscillatory squeeze flow using a micro-Fourier rheometer. Their results provided experimental support for the theoretical model of ER fluids under squeeze proposed by Sproston et al. (1994a). El Wahed et al. (1999) reported an experimental investigation into the influence of particle size on the effectiveness of an ER fluid in dynamic squeeze flow. They demonstrated that small particles are generally the more effective in terms of the level of transmitted force if compared to large particles. Vieira et al. (2001) used a mechanical testing machine to investigate the mechanical properties of ER fluids under tensile, compressive, and oscillatory squeeze tests. Their results showed that ER fluids are more resistant to compressive than to tensile stress. In compression tests, the compressive stress increased with the increase of the electric field strength. However, the increase of the initial gap size and the approaching velocity produced a decrease on the compressive stress. Tian et al. (2002a) found that both the compressive stress and the compressive modulus in ER fluids show an exponential relationship with the compressive strain, while the strain is larger than 0.1. Stepwise compression experiments demonstrated that with the increase of applied voltage, ER fluids show a change of electric field-determined behavior to the plastic fluid compressive mode [Tian et al. (2002b)]. A comprehensive study of the volume fraction dependence in 
squeezed ER fluids has been traditionally impeded by the "sealing effect" appearing under constant area operation [McIntyre and Filisko (2010)]. These restrictions were avoided by using a constant volume apparatus [McIntyre and Filisko (2007)]. At high field strengths, low compression rates, and low viscosity of the continuous phase most of the particles are kept between the plates and only the dispersing phase is squeezed out [Lynch et al. (2006); McIntyre and Filisko (2010); Tian et al. (2010)]. This fact has been parametrized in the form of a Peclet number as the ratio of viscous or convective forces to diffusive terms [Collomb et al. (2004); McIntyre and Filisko (2010)]. Most of the squeeze flow literature reviewed above concerns ER fluids and involves constant voltage and constant area compression. In this case, the electric field strength and volume concentration increase during the process. Thus, any change in the compressive stress is associated to the change of the electric field, volume fraction, and structural strength (i.e., the so-called structure parameter [Conrad (1998); Wu and Conrad (1998)]). Even though this problem can be partially skipped in the case of tensile flow by using normalized methods [Tian and Zou (2003)] it is an important drawback. In principle, when a MR fluid is compressed under a constant volume in the presence of a dc uniform magnetic field, the change of compressive stress may indicate mostly the change of the structure parameter in the MR fluids.

Surprisingly, the squeeze flow behavior of MR fluids has not been studied so thoroughly compared with their ER analogs. First reports on this topic concern compressionassisted-aggregation processes to achieve high-efficiency MR fluids by the so-called squeeze-strengthening effect. This consists of enhancing the yield shear stress in fieldresponsive fluids by the formation of thick strong columns under compression [Tang et al. (2000)]. This approach was further corroborated by Zhang et al. (2004, 2009). Contrarily, See et al. (2006) presented experiments in a MCR (Anton Paar) rheometer where compression did not have a large effect in the shear response. A detailed comparative study of the behavior of MR fluids under steady shear flow and constant velocity squeezing flow was reported by See (2003). The measurements showed that the mechanical response under squeezing flow scaled as $H^{0.91}$, whereas the response under shearing scaled as $H^{1.4}$. A special device was designed and fabricated to perform squeeze, torsional, and squeeze combined with torsional flows on an Instron multiaxial testing machine [Kulkarni et al. (2003)]. Contrary to what is believed [Tang et al. (2000)], it was experimentally observed that the introduction of a squeeze component in shearing flows (oscillatory torsional mode) does not always increase the strength of the MR fluid [Kulkarni et al. (2003)]. Mazlan et al. (2008) described the effects of compressive speed and magnetic field strength in the stress-strain curves of commercial MR fluids. On one hand, they observed a negligible effect of the compressive speed. On the other hand, the higher the magnetic field, the faster the stress-strain curve developed.

\section{SQUEEZE FLOW THEORY}

Under squeeze flow a material is compressed between two parallel plates. A schematic representation is shown in Fig. 2. Here, the sample of volume $V$ is positioned between two circular plates initially separated a gap distance $h_{0}$. In this section we will look for the relationship between the normal force $F$, height of the sample layer $h$, and squeezing speed of the moving plate, $v$, as this dependency can be used to obtain important rheological properties of the sample and in particular its yield stress.

To investigate the squeeze flow properties the Reynolds number Re has to be first estimated. In this operation mode, the Reynolds number can be defined as $\operatorname{Re}=h_{0} v \rho / \eta$, where $\rho$ is the suspension density and $\eta$ is the suspension viscosity [Engmann et al. 


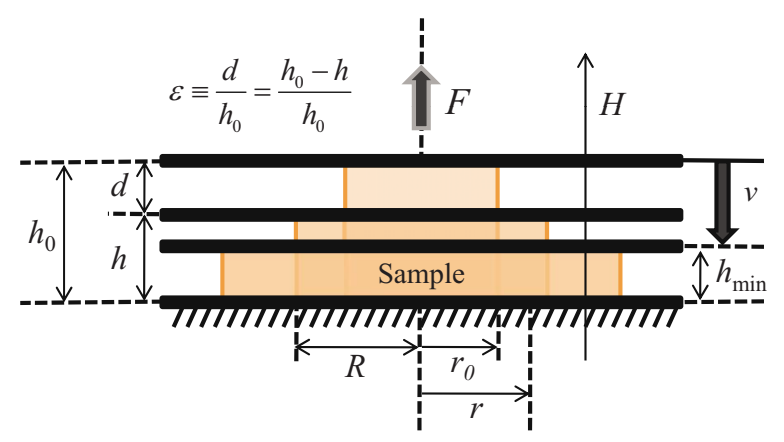

FIG. 2. Schematic diagram of the constant volume squeeze flow experiment (not to scale). For simplicity the free surface profile is represented as a vertical line. In a typical experiment $h_{0}=300 \mu \mathrm{m}$ and $h_{\min }=50 \mu \mathrm{m}$. The largest compressive strain was $\varepsilon \sim 0.83$.

(2005)]. The squeeze flow analysis is greatly simplified here because lubrication theory and creeping flow approximation can be invoked as $\operatorname{Re} \sim 10^{-3} \ll 1$.

\section{A. Newtonian fluids}

Since the pioneering work by Stefan (1874), the squeeze behavior of Newtonian fluids has received a lot of attention [e.g., Dienes and Klemm (1946); Campanella and Peleg (1987); Raphaelides and Gioldasi (2004)]. Under constant volume $V$ and no-slip condition Reynolds equation predicts the following radial pressure distribution (see Fig. 2):

$$
p(r)=\frac{3 \eta v}{h^{3}}\left[R^{2}-r^{2}\right]
$$

where $R$ is the radius of the fluid volume.

The normal force $F$ acting on the plate is simply obtained by integration on the surface:

$$
F=\frac{6 \pi \eta v}{h^{3}} \int_{0}^{R}\left[R^{2}-r^{2}\right] r d r=\frac{3 \pi \eta v}{2 h^{3}} R^{4},
$$

and therefore the so-called Stefan's equation results:

$$
F=\frac{3 \eta v V^{2}}{2 \pi h^{5}}
$$

Stefan's equation assumes a no-slip boundary condition at the plates surfaces. However, this fact does not always holds, and generally the presence of slip at the surfaces results in a significant force reduction when squeezing the sample [Engmann et al. (2005)]. Actually, analytical expressions exist in the literature for no-slip (i.e., "frictional" surfaces) [Dienes and Klemm (1946)] and perfect slip (i.e., "lubricated" surfaces) [Campanella and Peleg (1987); Raphaelides and Gioldasi (2004)], with the latter providing a lower limit for the forces with $F \sim h^{-2}$ in the case of constant volume operation. In most cases neither no-slip nor perfect slip occurs, and partial slip arises at the moving surface where the radial velocity at the surface $v_{r}$ takes on a linear form $v_{r}=v_{s} r / R$. Here, the slip parameter $v_{s}$ can be adjusted between no-slip at a minimum value and perfect slip at its maximum value; $0<v_{s}<v R / 2 h$. Several authors have investigated the partial slip situation in the past [Laun et al. (1999); McIntyre (2008)]. 


\section{B. Inelastic yield stress fluids: A continuum approach}

The squeeze flow behavior of inelastic yield stress fluids under no-slip conditions was initially studied by Scott (1929). The problem was later addressed by Covey and Stanmore (1981) who revisited it and reported theoretical and experimental investigations using a parallel-plate plastometer. Fluids obeying a Bingham constitutive equation were analyzed in their paper under both constant volume and constant radius operation regimes.

When dealing with yield stress fluids a plasticity number $S$ can be introduced [Covey and Stanmore (1981)]:

$$
S=\frac{\eta_{p} v R}{h^{2} \tau_{y}}
$$

The plasticity number separates two regimes. At low $S$ number the resistance to deformation arises from the yield shear stress component. However, at large $S$ number the major resistance to flow arises from viscous effects. Analytical expressions exist for the limits $S<0.05$ and $S>10$, whereas intermediate values of $S$ require numerical methods. In MR experiments presented in this work, $S$ was found to be always smaller than 0.05 whatever the gap distance and magnetic field strength applied, hence suggesting that particle aggregates will dominate the flow behavior.

Starting from the Cauchy-momentum equation for an incompressible fluid, Covey and Stanmore (1981) calculated the pressure on the fluid. Then, integrating the pressure over the total plate area gives a relation between the normal force $F$ and the approaching velocity $v$ :

$$
F=\frac{2 \pi \tau_{y} R^{3}}{3 h}+\frac{4 \pi}{7 h^{2}} \sqrt{2 \tau_{y} \eta_{p} v R^{7}}
$$

It must be noted here that Eq. (6) was originally obtained for constant radius experiments; however, change to the constant volume condition can be simply made by substituting $V=\pi R^{2} h$ [Covey and Stanmore (1981)].

Subsequent publications in this field demonstrated that theoretical work was in some conflict with regard to the flow pattern produced in the geometry [Lipscomb and Denn (1984)]. Moreover, the Bingham model was found to be unnecessarily complicated for numerical simulations. Gartling and Phan-Thien (1984) carried out a theoretical analysis that paralleled the method proposed by Covey and Stanmore (1981), this time using a biviscosity model instead of the Bingham description. This biviscosity model was later used by Williams et al. (1993) in the development of a time-dependent sinusoidal squeeze flow theory for ER fluids that was successfully applied to a prototype automotive engine mount.

According to the theory by Williams et al. (1993), initially developed for ER fluids and adapted here for MR fluids, the normal force acting on the plates can be expressed by the following equation:

$$
F=\frac{2 \pi \tau_{y} R^{3}}{h \chi^{3}}\left[\frac{\gamma^{3}}{108}+\int_{\gamma / 3}^{\chi} S^{2} G d S\right]
$$

where $\gamma, \chi, S$, and $G$ are parameters defined by 


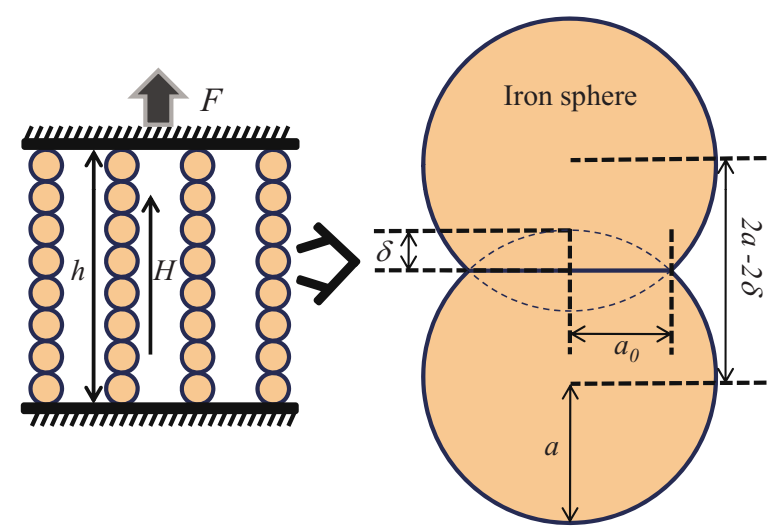

FIG. 3. Schematic representation illustrating the standard micromechanical model consisting in a cubic network of single chains.

$$
\gamma=\frac{\eta}{\eta_{r}}, \quad S=\frac{\eta v r}{h^{2} \tau_{y}}, \quad \chi=S(r=R), \quad G=-\frac{h}{2 \tau_{y}} \frac{d p}{d r} .
$$

Here, $\eta_{r}$ is the preyield viscosity and $\eta$ is the viscosity above the yield point in the biviscous model.

Interestingly, when the plasticity number $S$ is small, pressure gradient $G$ and thus the compressive force are driven by the yield shear stress and the viscous contribution is negligible. Under this condition, Eq. (7) is simplified to [Meng and Filisko (2005)]

$$
F=\frac{2 \pi \tau_{y} R^{3}}{3 h}
$$

which also corresponds to the low $S$ limit of Eq. (6) that was obtained from a Bingham approach. This finding allows us to conclude that both macroscopic descriptions, involving Bingham or biviscous models, result in a normal force that varies with the gap distance as $h^{-5 / 2}$ under constant volume operation.

Whereas the theory presented above treats field-responsive fluids as homogeneous materials and model them as simplified Bingham [cf. Eq. (6)] or biviscous fluids [cf. Eq. (7)], in reality, field-responsive fluids are two-phase fluids consisting of particle aggregates immersed in a continuous liquid phase. In the next section we provide a squeeze flow theory under a simplified microscopic description.

\section{Dilute MR fluids: A microscopic description}

Yang first studied the compressive and tensile behavior of dilute ER fluids by the electrostatic polarization model and Hertzian contact theory [Yang (1997)]. In this section we will adapt Yang's theory to the case of MR fluids.

A first microscopic insight in the normal force versus gap dependence of dilute MR fluids can be obtained under the assumption that particles aggregate to form single chains of particles aligned with the field (see Fig. 3). With this, the gap thickness $h$ can be expressed as

$$
h=2 N_{p c}(a-\delta),
$$

and the normal force $F$ acting on the plate is 


$$
F=N_{c} f
$$

Here, $N_{p c}$ is the number of particles in a chain, $a$ is the radius of the particle, $\delta$ is the indentation depth, $N_{c}$ is the number of chains, and $f$ is the force between the particles. Assuming that the particles behave as purely elastic materials, Hertz contact theory states that the radius of the contact area between the particles, $a_{0}$, is given by

$$
a_{0}^{3}=\frac{3 a}{4} \frac{1-\nu^{2}}{E}\left(f+f_{m}\right)
$$

where $\nu$ is the Poisson ratio, $E$ is Young's modulus, $f_{m}$ is the magnetostatic interaction force between the spheres, and the indentation depth is $\delta=a_{0}^{2} / a$.

As a first approximation, we can assume dipolar magnetic interactions for aligned dipoles. Hence,

$$
f_{m}=f_{0} \frac{a^{6}}{(a-\delta)^{4}} H^{2}
$$

where $f_{0}=(3 / 2) \pi \mu_{0} \mu_{c r} \beta^{2}$ and $\beta=\left(\mu_{p r}-\mu_{c r}\right) /\left(\mu_{p r}+2 \mu_{c r}\right)$. Here, $\mu_{0}$ is the permeability of vacuum, $\mu_{p r}$ is the relative permeability of the particles, $\mu_{c r}$ is the relative permeability of the continuous phase, $\beta$ is the magnetic contrast factor, and $H$ is the magnetic field strength.

After some algebra, combining Eqs. (10)-(13) we arrive at

$$
F=\frac{3}{2} \frac{\phi V f_{0} H^{2}}{\pi h}\left[\frac{4 E}{3 f_{0} H^{2}\left(1-\nu^{2}\right)}\left(\frac{\delta}{a}\right)^{3 / 2}\left(1-\frac{\delta}{a}\right)-\left(1-\frac{\delta}{a}\right)^{-3}\right] .
$$

Interestingly, the force-gap dependence scales with a power of -1 . This is a significantly weaker gap distance dependence if compared to predictions obtained from macroscopic models [cf. Eq. (9)]. As observed, the sustainable load increases nonlinearly with decreasing separation of the surfaces and is a linear function of the elastic modulus of the particles.

\section{EXPERIMENT: SQUEEZE FLOW MAGNETORHEOMETRY}

\section{A. Apparatus}

A MCR 501 (Anton Paar) magnetorheometer was employed to investigate the squeeze flow MR behavior. Nonmagnetic (titanium-based) parallel plates of diameter $20 \mathrm{~mm}$ were used. A scheme of the experimental squeeze flow test is sketched in Fig. 2. In the compression tests the original commanded gap was set as $h_{0}=300 \mu \mathrm{m}$, and then the upper plate moved slowly down toward the stationary bottom plate at a constant velocity $v$, while the normal force acting on the upper plate was monitored. The measuring range of the force sensor is $\pm 50 \mathrm{~N}$, and its compliance is $0.59 \mu \mathrm{m} / \mathrm{N}$. The distortion of the force sensor under the pressures handled in this work was neglected. As a measure of the deformation, the compressive strain was defined here as $\varepsilon=\left(h_{0}-h\right) / h_{0}=d / h_{0}$. Typically, in our experiments the maximum compressive strain achieved was $\varepsilon \sim 0.8$, while the strain rate was around $\dot{\varepsilon} \sim 0.03-0.2 \mathrm{~s}^{-1}$.

An external magnetic circuit was used to generate uniaxial dc magnetic fields of the order of $\sim 100 \mathrm{kA} / \mathrm{m}$ in the gap between the plates [Wollny et al. (2002)]. Due to geometrical constrictions, a central hole exists to pass the rheometer shaft through. As a consequence, the magnetic field is not absolutely uniform in the vertical and radial directions [Laeuger et al. (2005); Laun et al. (2008)]. This may result in some particle migration toward the rim of the plates where the magnetic field is stronger, especially at 
the largest field strengths investigated [Andablo-Reyes et al. (2011); de Vicente et al. (2009)]. This migration enhances local magnetization at the rim, thus further increasing the magnitude of the flux density maximum [Laun et al. (2008)]. In order to achieve a reasonably uniform radial magnetic flux density profile, we studied the effect of sufficiently small magnetic field strengths $(\leq 354 \mathrm{kA} / \mathrm{m})$ on dilute MR fluids $(5 \mathrm{vol} \%)$. Under this situation, the density profiles are expected to be rather uniform in the volume occupied by the sample as observed in Fig. 5 of Laun et al. (2008). Magnetic field strengths reported in this paper refer to the maximum plateau value corresponding to radial magnetic flux density profiles provided by the manufactures of the magnetocell [Fig. 3 of Laeuger et al. (2005)]. It should also be remarked that preliminary tests were carried out on nonmagnetic samples to confirm that the application of the magnetic field did not result in a measurable normal force. It should also be stressed that the magnetic field density in the MR fluid changes upon changing the gap since the magnetic resistance associated to the gap is modified. However, this fact has not been addressed in the present analysis. The plates were assumed to be perfectly parallel even though a small misalignment is feasible [Andablo-Reyes et al. (2010)]. Nonetheless, our experiments did not reach strains larger than $\varepsilon=0.8$ which helped preventing a serious error caused by the nonparallelism of the plates. The gap error was accounted for by calibration using a semiempirical approach that involves running measurements on Newtonian fluids at different gap distances. In particular, following the method by Connelly and Greener (1985) a gap error of $17 \mu \mathrm{m}$ was estimated in our plate-plate geometry.

\section{B. Materials preparation and experimental protocol}

Newtonian fluids used in this study were silicone oils having different viscosities (100 and $350 \mathrm{mPa} \mathrm{s}$ ). These were obtained from Sigma-Aldrich and used without further purification. MR fluids were prepared by carefully mixing carbonyl iron powder (HQ, BASF; diameter of $\sim 800 \mathrm{~nm}$ ) in silicone oil (20 mPa s, Sigma-Aldrich) to get suspensions having a volume fraction of 5 vol $\%$ (metallic iron density of $7.8 \mathrm{~g} / \mathrm{cm}^{3}$ ). This concentration is small enough for the models to be applied [cf. Sec. III C] and large enough to get a measurable normal force signal. HQ carbonyl iron particles were chosen for this study because they are used in the formulation of the vast majority of MR fluids reported in the literature [Park et al. (2010); de Vicente et al. (2011) and references therein]. The preparation of suspensions consisted of the following steps: (i) magnetic powder and silicone oil were mixed in a polyethylene container; (ii) the mixture was stirred first by hand, and then in an ultrasonic bath; and (iii) step (ii) was repeated several times to ensure the required final homogeneity.

Basically, two possibilities exist when studying the squeeze behavior of a material: constant area and constant volume approaches. Here, the tests were carried out under constant volume conditions. This means that, a priori, the area of the plate in contact with the sample changes accordingly to the change in gap thickness. With this, the concentration of the particles that stay in between the plates is known throughout the experiment in contrast to what happens for ER fluids under conventional constant area experiments because of the so-called sealing effect. Another advantage of using constant volume tests is the fact that inertial effects are minimized contrarily to constant area experiments where corrections are usually needed [McIntyre (2008); McIntyre and Filisko (2010)].

Generally speaking, squeeze flow tests are typically run under constant normal force applied $F$ or constant approaching velocity $v$ (i.e., constant rate loading/compression). The experiments presented in this paper concern constant approaching speed investigations $(1-10 \mu \mathrm{m} / \mathrm{s})$ in a displacement control mode resulting in nonsteady tests with 
variable strain rate. In this case, the inertial terms contained in the force equation vanish [Bird (1987)]. Furthermore, the small approaching speeds used guarantee that results presented here corresponded to low $S$ numbers [Covey and Stanmore (1981)] and to the so-called filtration regime of operation [McIntyre and Filisko (2010)].

In a typical experiment a small amount of the MR fluid (initial radius $r_{0}$ $=3.8 \pm 0.3 \mathrm{~mm}$ and volume $V \sim 14 \mu \mathrm{l}$ ) was deposited on top of the lower plate using a microliter syringe (MV17990 Boeco, Germany). Once the sample had relaxed the normal force transducer was reset to zero. Next, a uniaxial dc magnetic field was suddenly applied across the plates with the sample subjected to no deformation. This period was long enough to allow the aggregates to form. After $60 \mathrm{~s}$, the compression test was started at a constant approaching speed $v$ still in the presence of the magnetic field. Results presented below are always averages over at least three separate runs with fresh new samples. All experiments were run at $25{ }^{\circ} \mathrm{C}$. It is worth to remark that the normal force did not experience any measurable variation when the magnetic field was applied in the quiescent state due to the small particle concentration in the MR fluid. Finally, the way how to increase the magnetic field, either suddenly or gradually, was also checked and was found not to be important in the compression test results.

\section{PARTICLE-LEVEL SIMULATIONS}

Three-dimensional (3D) MR simulations were carried out following a simplified method developed by Klingenberg and co-workers [Klingenberg et al. (1989); Klingenberg et al. (1991); Heine et al. (2006)]. The MR fluid was modeled as a suspension of $N$ buoyant inertialess particles in a Newtonian fluid of viscosity $\eta_{c}$ and relative magnetic permeability $\mu_{c r}$. The particles were considered to be monodisperse with diameter $2 a$ and relative magnetic permeability $\mu_{p r}$. When a magnetic field is applied the particles are magnetized due to their difference of permeability with respect to the continuum medium. Assuming pairwise additivity and taking the point dipole approximation, the magnetic force exerted on the particle $i$ by the rest of the particles is

$$
\begin{gathered}
\vec{F}_{i}^{m}=\sum_{j=1, j \neq i}^{N} F_{0}\left(\frac{2 a}{r_{i j}}\right)^{4}\left[\left(3 \cos ^{2} \theta_{i j}-1\right) \hat{r}+\sin \left(2 \theta_{i j}\right) \hat{\theta}\right], \\
F_{0}=\frac{3}{4} \pi \mu_{0} \mu_{c r} \beta^{2} a^{2} H^{2},
\end{gathered}
$$

where $F_{0}$ is the so-called magnetic field dependent constant; $\hat{r}$ and $\hat{\theta}$ are the unit vectors parallel and perpendicular to the line joining the pair of particles, respectively; $r_{i j}$ is the distance between the centers of particles $i$ and $j$; and $\theta_{i j}$ is the angle between the vectors joining the centers of particles $i$ and $j$ and the external magnetic field $H$ (Fig. 4). In contrast to previous simulations on ER fluids, where the voltage is kept constant and the electric field strength increases upon decreasing the gap, here $H$ is assumed to be uniform and constant while compressing the fluid. Multibody and multipolar interactions are neglected.

To prevent the particles from overlapping we introduced a short-range repulsive exponential interaction force: 


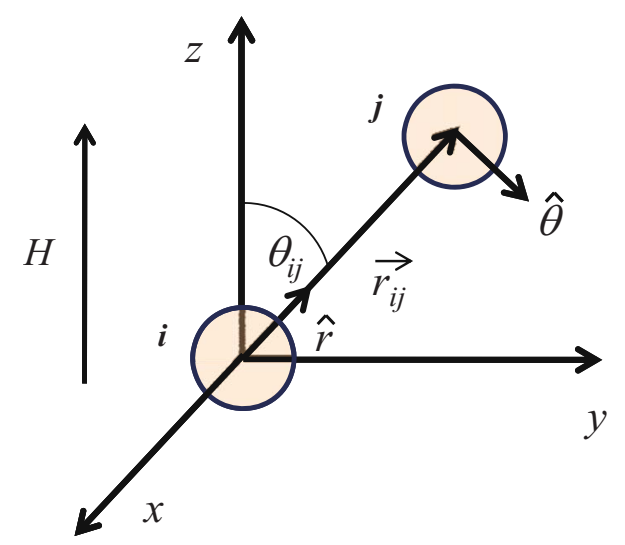

FIG. 4. Schematic representation of the coordinate reference system used in particle-level simulations.

$$
\vec{F}_{i}^{r}=-\sum_{j=1, j \neq i}^{N} F_{0} \exp \left[-100\left(\frac{r_{i j}}{2 a}-1\right)\right] \hat{r} .
$$

The particles were also subjected to a drag viscous force. In the free draining approximation the hydrodynamic force on particle $i$ is given by

$$
\vec{F}_{i}^{h}=-6 \pi \eta_{c} a\left(\frac{d \vec{r}_{i}}{d t}-\vec{u}_{i}^{\infty}\right),
$$

where $\vec{u}_{i}^{\infty}$ stands for the laminar viscous fluid velocity at the center of particle $i$. For squeeze flow under no-slip boundary conditions in the limit of creeping flow $(\operatorname{Re} \rightarrow 0)$, the velocity field is given by [Kim et al. (1999)]

$$
\vec{u}_{i}^{\infty}=\left(\frac{3 r_{i} z_{i} v}{h^{2}}\right)\left(1-\frac{z_{i}}{h}\right) \hat{r}-3 v\left(\frac{z_{i}}{h}\right)^{2}\left(1-\frac{2}{3} \frac{z_{i}}{h}\right) \hat{z},
$$

where $r_{i}$ and $z_{i}$ are the cylindrical coordinates of particle $i$.

The particles were confined between two walls, represented by two rigid and parallel planes positioned at $z=0$ and $z=h$. Again, to prevent particles from penetrating into the plates we employed a short-ranged repulsive potential:

$$
\vec{F}_{i}^{w}=F_{0} \exp \left[-100\left(\frac{\xi_{i}^{l, u}}{2 a}-\frac{1}{2}\right)\right] \hat{n},
$$

where $\hat{n}$ is the normal vector to the walls and $\xi_{i}^{l}=z_{i}$ and $\xi_{i}^{u}=h-z_{i}$ are the distances between the particle and the lower and upper walls, respectively. Particles at a distance of $1.1 a$ from the plates are fixed to them.

The position of the lower plate $z=0$ was fixed and the system was compressed by moving the upper plate at a constant approaching speed $v$. Thus, the position of the upper wall $h(t)$ at the instant $t_{i+1}=t_{i}+\Delta t$ was calculated as follows:

$$
h\left(t_{i+1}\right)=h\left(t_{i}\right)-v \Delta t .
$$

From the force expressions reported above arise the natural length, force, and time scales: $l_{s}=2 a, F_{s}=F_{0}$, and $t_{s}=12 \pi \eta_{c} a^{2} / F_{0}$, respectively. Hence, for inertialess particles, neglecting Brownian forces, the equation of motion for particle $i$ can be written as 


$$
\frac{d \vec{r}_{i}^{*}}{d t^{*}}=\vec{F}_{i}^{m *}+\vec{F}_{i}^{r *}+\vec{F}_{i}^{w *}+\vec{u}_{i}^{\infty * *}
$$

where dimensionless variables are denoted with an asterisk.

The equations of motion were integrated numerically using an explicit Euler method with a dimensionless time step $\Delta t^{*}=5 \times 10^{-4}$. Using a smaller time step had a negligible effect. On one hand, interparticle exponential repulsive interactions were evaluated for sphere separations less than a cutoff radius $4 a$. On the other hand, wall-particle repulsive interactions were evaluated only for a perpendicular distance from wall to particle center smaller than $3 a$. A further increase of the previously mentioned distances had no significant influence on the results. Periodic boundary conditions were not applied.

Experiments reported in this paper concern filtration-dominated regimes (low compression rates and low viscosity of the continuous phase). In this situation, most of the particles are kept between the plates, the structure is basically preserved, and only the dispersing phase is squeezed out as the plasticity number is small. As a consequence, as a first approximation, only magnetic forces are accounted for in the calculation of the normal forces. Hence, the normal force $F^{*}$ acting on the upper plate was calculated from the normalized total magnetic energy $U^{*}$,

$$
U^{*}=\sum_{i=1}^{N} \sum_{j=1, j \neq 1}^{N}\left(\frac{1}{r_{i j}^{*}}\right)^{3}\left(1-3 \cos ^{2} \theta_{i j}\right),
$$

according to

$$
F^{*}=-\frac{d U^{*}}{d h^{*}}
$$

It is worth to remark here that other approaches exist in the literature for the stress transfer determination between the system and bounding plates [Lukkarinen and Kaski (1996)].

When compressing any field-responsive colloid it is important to distinguish between the normal force contributions coming from the number of percolating field-induced clusters, $N_{c}$, and their internal structure. Basically, it is not only the number of percolating structures that contributes to the normal force, but also their thickness and strength. For this purpose we define a new parameter $\Pi_{i}$, for every percolating aggregate $i$ in the system, as follows:

$$
\Pi_{i}=\frac{N_{p i} h_{0}}{h},
$$

where $N_{p i}$ is the number of particles contained in the percolating cluster $i, h_{0}$ is the initial separation between the plates, and $h$ is the plate separation at time $t$. For every gap an averaged value can be calculated using the following expression:

$$
\Pi=\frac{\sum_{i=1}^{N_{c}} \Pi_{i}}{N_{c}}=\frac{\sum_{i=1}^{N_{c}} \frac{N_{p i} h_{0}}{h}}{N_{c}} .
$$




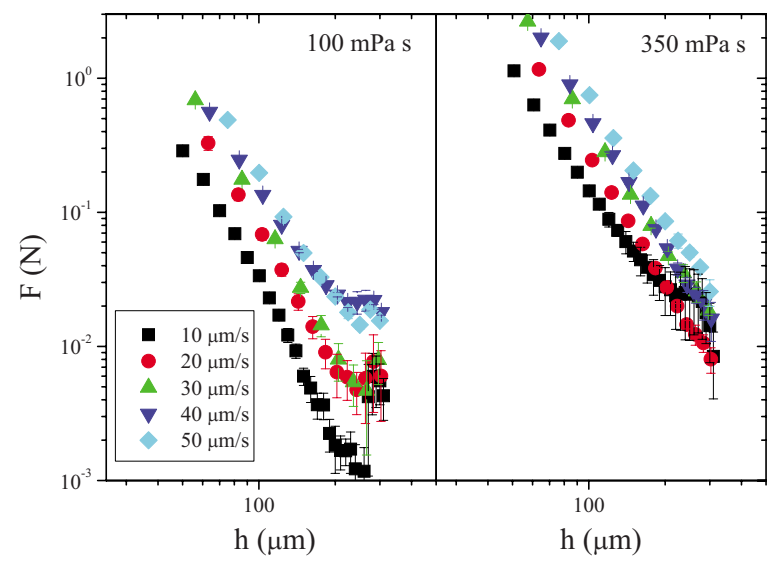

FIG. 5. Normal force $F$ versus gap distance $h$ at various approaching speeds for two Newtonian silicone oils.

\section{RESULTS AND DISCUSSION}

\section{A. Squeeze flow of Newtonian fluids}

Figure 5 shows the normal compressive force $F$ versus gap distance $h$ for Newtonian silicone oils (100 and $350 \mathrm{mPa} \mathrm{s}$ ) by moving the upper plate at a constant speed in the range $v=10-50 \mu \mathrm{m} / \mathrm{s}$, while the lower plate remained stationary. Experiments correspond to constant volume tests to make it possible a straightforward comparative study with MR fluids. As expected from the lubrication theory, in all cases investigated, the relationship between the force and gap follows a power law behavior. At very large gap separations (early stages of the compression experiment) data are not reliable because of the transient regime where some inertia may artifact the data.

The approaching speed does not seem to have any clear effect in changing the slope; an average slope is obtained that is around -3.2 well in the range $[-5,-2]$, corresponding to the behavior of Newtonian fluids under constant volume approach and in agreement with the literature predictions (see Sec. III A). It seems that the only effect of changing the approaching speed is shifting the curves in the vertical direction. As anticipated, the larger the viscosity, the larger the normal forces obtained.

Constant volume squeeze flow (no-slip and perfect slip) theories for Newtonian fluids suggest that experiments for different viscosity fluids and approaching speeds can be collapsed in a master curve if plotting the ratio $F / \eta v$ versus $h$ [Stefan (1874), Dienes and Klemm (1946); Campanella and Peleg (1987); Raphaelides and Gioldasi (2004)]. As observed in Fig. 6, a good collapse is obtained especially at narrow gaps where the lubrication approximation holds. No-slip Stefan equation does qualitatively explain the trend observed. However, the smaller than -5 slope observed may be ascribed to the presence of some partial slip. As is generally found, Stefan's equation with no-slip gives an exponent that is too high, and perfect slip gives an exponent that is too low.

The relationship between force and gap distance is essential in all squeeze flow deformations. Nevertheless, it is generally more convenient to use related magnitudes such as the compressive strain, defined here as the ratio of the moving distance of the upper plate to the initial distance between the plates $\varepsilon=d / h_{0}$, to make a straightforward comparative study with the rheological behavior under different standard flow conditions such as direct-shear and pressure-driven flows. We will use this description in the next section which is devoted to MR fluids. 


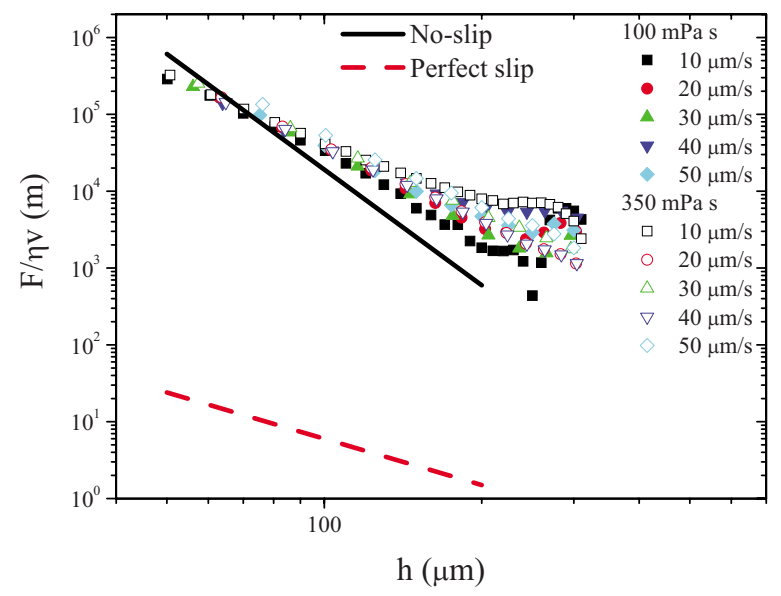

FIG. 6. Compressive force $F$ from Fig. 5 divided by the viscosity $\eta$ and approaching speed $v$ as a function of gap distance $h$. Lines correspond to Stefan's model predictions under no-slip and perfect slip conditions. The experimental error is contained within the symbol size.

\section{B. Squeeze flow of MR fluids}

\section{Unidirectional monotonic compression tests}

Experiments were carried out for a wide range of magnetic field strengths up to 354 $\mathrm{kA} / \mathrm{m}$. Magnetic fields investigated were high enough to discard the normal force contribution coming from the pure oil which is expected to be of only $\sim 10^{-3} \mathrm{~N}$ (cf. Fig. 6). Negligible differences were observed for the two approaching speeds investigated ( 1 and $10 \mu \mathrm{m} / \mathrm{s}$ ) in agreement with experimental data reported by Mazlan et al. (2008). This is possibly due to the small plasticity number [Eq. (5)], thus allowing the particles to find a minimum energy configuration in a well organized field-induced structure [Lukkarinen and Kaski (1996)]. It is worth to point out that no-slip at the surfaces was checked by using plates previously coated with a thin layer of carbonyl iron powder. Experiments that were carried out using these plates were coincident with those obtained using conventional noncoated titanium plates.

For the sake of brevity, in Fig. 7 we only illustrate results for an approaching speed of $10 \mu \mathrm{m} / \mathrm{s}$. Here, we show the influence of the dc magnetic field strength on the normal force-compressive strain curves for a 5 vol \% MR fluid. As observed, the force increases as we increase the magnetic field applied. This is anticipated to be a consequence of the MR effect. It is well known that under slow compressive strains $(\varepsilon \lesssim 0.05)$ MR fluids withstand the load without "breaking" (data not shown in Fig. 7 because of the limited strain resolution). Upon increasing the strain the squeeze flow behavior is expected to largely depend on the repeated formation/breakdown process of microscopic and macroscopic structures [Lukkarinen and Kaski (1998)]. Hence, structure breakdown occurs for $\varepsilon \sim 0.05$, and the subsequent steadily increasing force suggests that the metastable fieldinduced structure partially recovers from compression. It has been documented in the literature that for very rapidly compressed systems, the force decreases just after breakdown of the structure, suggesting that in this case the particles do not have time to recover. A rough estimation of the so-called plastic modulus increase under compression can be obtained by the ratio of the compressive stress and the associated true strain $\varepsilon_{t}$ $=\ln \left(h / h_{0}\right)$ [Monkman (1995)]. Taking $H=354 \mathrm{kA} / \mathrm{m}$ and $v=10 \mu \mathrm{m} / \mathrm{s}$ we estimated a change of approximately one order of magnitude from 2 to $62 \mathrm{kPa}$. Curves presented in 


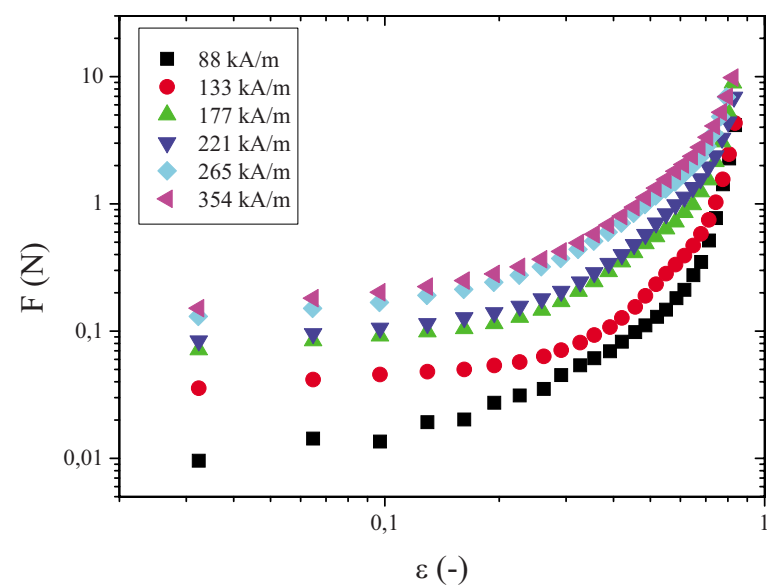

FIG. 7. Typical normal force $F$ versus compressive strain $\varepsilon$ curves of MR fluids at different magnetic field strengths $H(5$ vol \% MR fluid, $v=10 \mu \mathrm{m} / \mathrm{s})$.

Fig. 7 are very similar to stress-strain curves for ER fluids under constant area tests by Vieira et al. (2001). Moreover, our experimental results are in qualitative agreement with slow rate dynamics simulations by Lukkarinen and Kaski (1996) in the range $\varepsilon=0-0.25$ (see Fig. 2 in their letter).

\section{Low-strain normal force plateau}

According to Bingham and biviscous macroscopic models [cf. Eq. (9)], the normal force is expected to vary as $F=A /(1-\varepsilon)^{5 / 2}$. Here, $A$ represents the low-strain normal force and is associated to the yield compressive stress (see below). Experimental data shown in Fig. 7 were fitted to this equation, and fitting parameters $A_{E x p}$ are reported in Table I. To make possible a straightforward comparative study with macroscopic theoretical predictions, steady shear flow experiments were also carried out for a gap distance of $h_{0}=300 \mu \mathrm{m}$ with $5 \mathrm{vol} \%$ MR fluids (results not shown here for brevity). Static yield shear stresses $\tau_{y s}$ were determined from extrapolation in shear stress versus shear-rate

TABLE I. Fitting parameter $A_{E x p}$ for normal force-compressive strain curves reported in Fig. 7 according to $F=A /(1-\varepsilon)^{5 / 2}$. $A_{\text {Macro }}$ corresponds to macroscopic model predictions [Eq. (9)] using the static yield shear stress as an input. In these calculations we assumed $r_{0}=3.8 \mathrm{~mm}$ as visually determined. $\delta / a$ corresponds to the indentation depth calculated by fitting Eq. (14) to the experimental $A_{E x p}$ values. In these calculations we have taken $\nu=0.29$ and $E=211 \times 10^{9} \mathrm{~Pa}$ which correspond to typical values for pure iron.

\begin{tabular}{cccc}
\hline \hline $\begin{array}{c}\mathrm{H} \\
(\mathrm{kA} / \mathrm{m})\end{array}$ & $\begin{array}{c}A_{\text {Exp }} \\
(\mathrm{N})\end{array}$ & $\begin{array}{c}A_{\text {Macro }} \\
(\mathrm{N})\end{array}$ & $\begin{array}{c}\delta / a\left(\times 10^{-4}\right) \\
(-)\end{array}$ \\
\hline 88 & $0.0156 \pm 0.0018$ & 0.025 & 1.208 \\
133 & $0.0406 \pm 0.0012$ & 0.059 & 1.611 \\
177 & $0.084 \pm 0.003$ & 0.103 & 1.945 \\
221 & $0.099 \pm 0.005$ & 0.153 & 2.175 \\
265 & $0.163 \pm 0.010$ & 0.227 & 2.424 \\
354 & $0.192 \pm 0.012$ & 0.311 & 2.700 \\
\hline \hline
\end{tabular}


TABLE II. Suspension relative magnetic permeability $\mu_{s r}$ and magnetic contrast factor $\beta$ calculated using Maxwell-Garnett and FröhlichKennelly equations (Jiles, 1991).

\begin{tabular}{ccc}
\hline \hline $\begin{array}{c}\mathrm{H} \\
(\mathrm{kA} / \mathrm{m})\end{array}$ & $\begin{array}{c}\mu_{s r} \\
(-)\end{array}$ & $\begin{array}{c}\beta \\
(-)\end{array}$ \\
\hline 88 & 1.125 & 0.802 \\
133 & 1.117 & 0.751 \\
177 & 1.110 & 0.706 \\
221 & 1.103 & 0.665 \\
265 & 1.098 & 0.630 \\
354 & 1.088 & 0.568 \\
\hline \hline
\end{tabular}

ramp-up curves following the standard procedure [de Vicente et al. (2010)]. With this, a theoretical prediction for the constant $A$ is obtained under a macroscopic description along with $A_{\text {Macro }}=\left(2 \pi \tau_{y s} r_{0}^{3}\right) /\left(3 h_{0}\right)$ [cf. Eq. (9)]. Results are also included in the third column of Table I. Macroscopic theories slightly overestimate experimental results. However, the agreement is quite good bearing in mind that there are no free parameters. The discrepancy with experimental data may be explained because of the strong cubic dependence with the sample radius and the pipette error when depositing the drop on the rheometer plate.

The microscopic model described in Sec. III C qualitatively captures the low-strain normal force plateau as well. A quantitative theoretical estimation can also be obtained as follows. Since the particle volume fraction investigated here was significantly low, as a first approximation the internal magnetic field could be assumed to be simply the applied external magnetic field. As a consequence, the relative suspension permeability $\mu_{s r}$ and the contrast factor $\beta$ can be calculated straightforwardly from Maxwell-Garnett and Fröhlich-Kennelly equations [Jiles (1991)] for the magnetization-magnetic field strength dependence for the particles where the relative initial permeability of the solid phase is 40 and their saturation magnetization is $1550 \mathrm{kA} / \mathrm{m}$ [de Vicente et al. (2010)]. Results for $\mu_{s r}$ and $\beta$ for a range of magnetic fields investigated are shown in Table II. With these, the microscopic model prediction can be fitted to the experimental $A_{\text {Exp }}$ parameter resulting in a field dependent indentation depth $\delta / a$. The indentation depth is tabulated in the fourth column in Table I and increases when increasing the magnetic field. Some work is currently done in this direction to check whether these indentation values can be experimentally ascertained.

One of the most relevant rheological properties of a MR fluid is the yield stress that must be overcome to initiate gross material deformation or flow. The yield stress is associated to the minimum stress value required for the onset of flow and is known to be strongly dependent on the mode of operation. Under compression, the so-called yield compressive stress can be determined from the low-strain normal force plateau. The low-strain normal force plateau $A$ is expected to mimic the strength of the initial fieldinduced structure. In fact, it increases with the magnetic field strength following a power law function with exponent $1.89 \pm 0.25$, in good agreement with an exponent of 2 predicted by dipolar magnetostatic models [Bossis et al. (2002)]. The yield compressive stress can be estimated by the ratio between the low-strain normal force plateau and the wetted plate surface area. A comparative study on the yielding properties under shear and compression is shown in Fig. 8. Here, we show both the dynamic and static yield shear stresses as well as the yield compressive stress for MR fluids having 5 vol \% concen- 


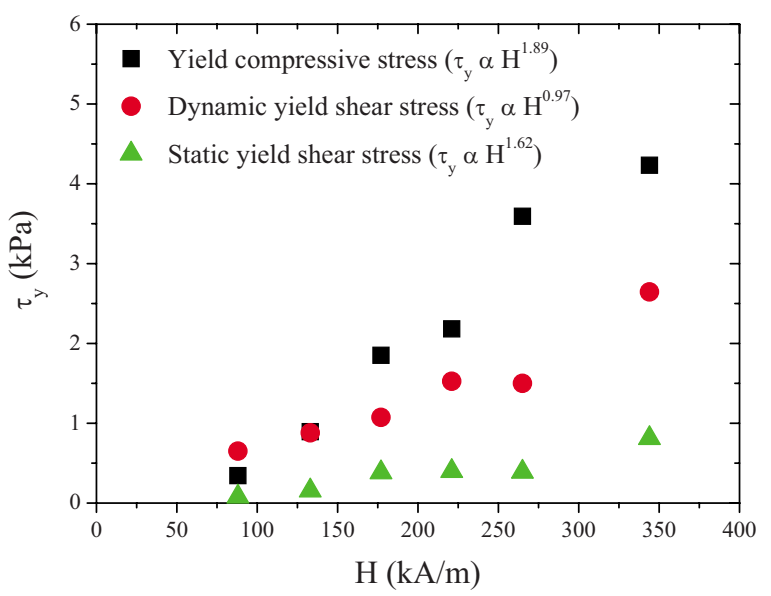

FIG. 8. Comparison between yield compressive stresses and yield shear stresses for 5 vol $\%$ MR fluids. For the calculation of the compressive stress we have assumed an initial radius of $r_{0}=3.8 \mathrm{~mm}$. For completeness we also show results for dynamic yield shear stresses obtained by extrapolation in lin-lin representations of shear stress versus shear rate at large deformations (Bossis et al., 2002).

tration. It is observed that the yield compressive stress is significantly larger than the yield shear stresses, in good agreement with other experiments reported in the literature (see Sec. II). Even though dynamic yield stress is observed to be larger than the static yield stress in Fig. 8, some ER systems have been reported in the past to exhibit opposite trends [Kim et al. (2007)].

To sum up, Bingham, biviscous, and micromechanical models do satisfactorily explain the existence of a yielding low-strain normal force plateau under constant volume compression. In the case of macroscopic models, a reasonably good agreement exists when comparing theoretical and experimental results. The next question to answer is whether these models suffice to explain the flow behavior at larger compressive strains.

\section{Compressive flow behavior}

Interestingly, experimental results presented in Fig. 7 can be reasonably collapsed when dividing by the low-strain normal force as shown in Fig. 9. As included in Fig. 9 we show theoretical predictions from macroscopic [Eq. (9)] and microscopic [Eq. (14)] models. As observed, the microscopic theoretical curve (dashed line) stays below the experimental data. As expected, this model does not conveniently capture the force-strain dependence at intermediate and large strain values as it simply assumes that only single chains of particles exist under flow behavior. On the contrary, a much better agreement is observed for the macroscopic prediction (solid line). In this case, the slightly faster than theoretically expected increase of the normal force can be qualitatively explained under the framework of Eq. (9) in terms of a yield shear stress that exponentially increases with the compressive strain (dotted line). We will go back to this point in Sec. VI B 4.

Similarly to Tian et al. (2002a), the intermediate strain region $(0.2<\varepsilon<0.5)$ is also satisfactorily captured by an exponential function $F=\alpha_{1} \mathrm{e}^{\alpha_{2} \varepsilon}$ (fitting parameters contained in Table III). On one hand, $\alpha_{1}$ values were found to increase with the magnetic field strength. On the other hand, $\alpha_{2}$ values were approximately constant and very close to experiments reported by Tian et al. (2002a) for ER fluids under constant area and variable electric field strength (constant voltage). The physical meaning of this exponential relationship is not clear yet by simply observing normal force changes during compression. 


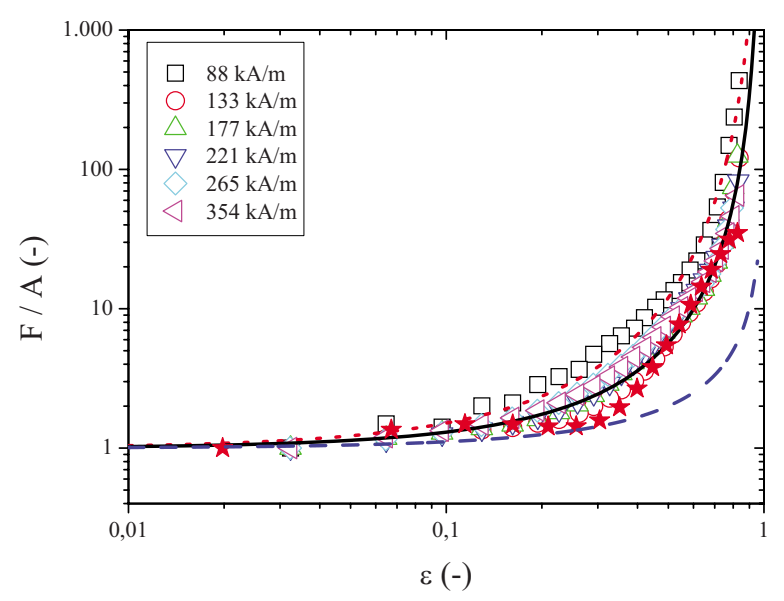

FIG. 9. Dimensionless normal force $F$ as a function of compressive strain $\varepsilon$ for different magnetic fields. Blue dashed line corresponds to Eq. (14); $F / A=1 /(1-\varepsilon)$. Black solid line corresponds to Eq. $(9) ; F / A=1 /(1$ $-\varepsilon)^{5 / 2}$. Red dotted line corresponds to the expression $F / A=\mathrm{e}^{1.5 \varepsilon} /(1-\varepsilon)^{5 / 2}$. Red stars represent particle-level simulation results.

As reported above, when using a low viscosity continuous medium with a low squeezing speed the viscous contribution to the normal force is expected to be minimal. As the plates compress the structures, the columns become shorter and thicker as some particles break away and reform the structure [McIntyre (2008)]. These reformed structures are actually stronger than the original and hence is expected a squeeze-driven strengthening effect [Tang et al. (2000)], eventually resulting in a yield shear stress that increases with the compressive strain. In order to get a better understanding of the structural evolution under compression we now perform combined flow tests.

\section{Squeeze combined with torsional small-amplitude oscillatory flow}

To further investigate the structural changes during squeeze, we carried out compression tests by superposition of a SAOS flow where the upper plate was made to undergo rotary oscillations about a mean position $\left(5 \times 10^{-6} \mathrm{rad}\right)$ at a given frequency $(f$ $=10 \mathrm{~Hz}$ ) in the viscoelastic linear region. This kind of test would allow us to monitor viscoelasticity changes at the same time the sample is being compressed. It is worth to

TABLE III. Fitting parameters $\alpha_{t}$ and $\alpha_{2}$ for normal force-compressive strain curves reported in Fig. 7 in accordance with $F=\alpha_{1} \mathrm{e}^{\alpha_{2} \varepsilon}$. The approaching speed is $v=10 \mu \mathrm{m} / \mathrm{s}$. The strain fitting range is $\varepsilon \sim 0.1-0.5$.

\begin{tabular}{ccc}
\hline \hline $\begin{array}{c}\mathrm{H} \\
(\mathrm{kA} / \mathrm{m})\end{array}$ & $\begin{array}{c}\alpha_{1} \\
(\mathrm{~N})\end{array}$ & $\begin{array}{c}\alpha_{2} \\
(-)\end{array}$ \\
\hline 88 & $0.0107 \pm 0.0004$ & $4.81 \pm 0.09$ \\
133 & $0.0174 \pm 0.0020$ & $4.90 \pm 0.26$ \\
177 & $0.0425 \pm 0.0019$ & $4.98 \pm 0.10$ \\
221 & $0.0463 \pm 0.0028$ & $5.21 \pm 0.13$ \\
265 & $0.0904 \pm 0.0021$ & $4.93 \pm 0.05$ \\
354 & $0.1030 \pm 0.0034$ & $4.91 \pm 0.07$ \\
\hline \hline
\end{tabular}


(a)
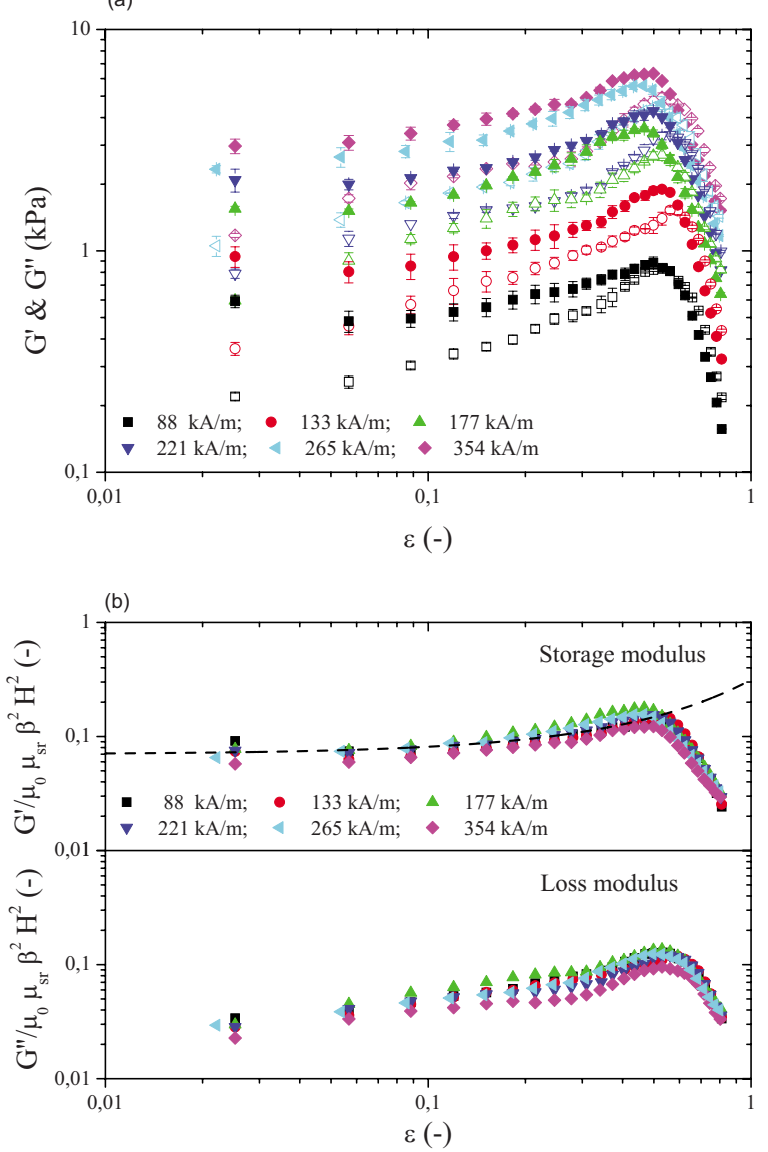

FIG. 10. Strain dependence of the shear viscoelastic moduli for different magnetic field strengths. (a) Storage and loss moduli as functions of compressive strain. Closed symbol, $G^{\prime}$; open symbol, $G^{\prime \prime}$. (b) Normalized storage and loss moduli as functions of strain. Dashed line corresponds to $G^{\prime} /\left(\mu_{0} \mu_{s r} \beta^{2} H^{2}\right)=0.07 \mathrm{e}^{1.5 \varepsilon}$.

remark here that a few works exist in the literature concerning combined flows in spite of their importance to get structural information. Relevant papers on this subject are those by Kulkarni et al. (2003) and See et al. (2006).

Preliminary tests were carried out to ensure that SAOS was not affecting the resulting normal force measured under compression. We obtained a very good reproducibility using different samples. The typical viscoelastic response is shown in Fig. 10(a) for an approaching speed of $v=10 \mu \mathrm{m} / \mathrm{s}$. We observe that $G^{\prime}$ initially increases with increasing the strain, in agreement with experiments carried out by See et al. (2006). Then, the viscoelastic moduli reach a peak at a gap thickness corresponding to $\varepsilon \sim 0.5$. These results suggest that when the MR fluid is squeezed, not only the compressive stress (as demonstrated above) but also the shear resistance increases with the gap reduction in an initial stage. However, for strains larger than $\varepsilon \sim 0.5$, the loss factor $\tan \delta=G^{\prime \prime} / G^{\prime}$ becomes 1, and then viscous shear flow occurs for larger strains. In Fig. 10(b) we show that the shear viscoelastic moduli scale with $\mu_{0} \mu_{s r} \beta^{2} H^{2}$, suggesting that dipolar magnetostatic interactions drive the microstructural evolution under compression. As anticipated in the section above, the squeeze-strengthening effect is found to roughly increase, following an 


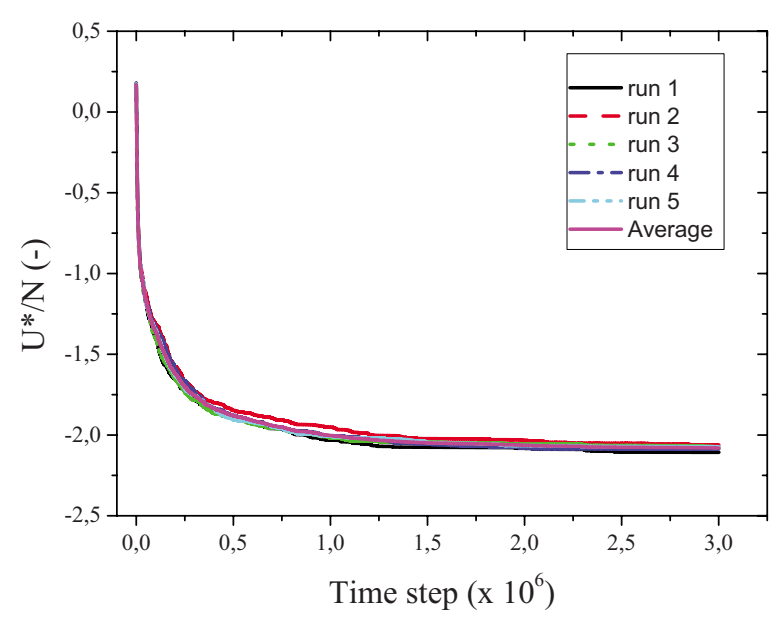

FIG. 11. Evolution of the magnetic energy normalized with the number of particles in the absence of flow for five different replicates; $h_{0}=40 a$.

exponential law as demonstrated by the dashed line in Fig. 10(b). This further supports the idea of a filtration-dominated regime and also the fact that the internal magnetic field is not significantly changing upon compression.

In summary, experiments presented above provide a complicated picture of the squeeze flow behavior of MR fluids under constant volume in the presence of dc magnetic fields. At very low strains structures are able to resist without breaking (results not shown here because of a technical limitation of our magnetorheometer). Upon increasing the compressive strain structures do eventually break. The behavior at larger strains is dictated by the balance between reformation and fragmentation processes. In the case of slow compression rates particles do have time to find a minimum energy configuration and structures reform under compression. This is manifested in an increase of the normal force and an enhancement of the shear storage and loss moduli. Interestingly, for very large strains normal forces do continue increasing. However, viscoelastic moduli stop growing entering in a region that is shear viscosity dominated.

Both macroscopic and microscopic models employed above suffer from important drawbacks at medium and large strains mostly due to the fact that the field-induced microstructure evolves under compression. A more direct approach involves the use of particle-level computer simulation techniques.

\section{Simulations}

Initially, 600 particles are placed in a cylindrical volume (radius of $48 a$ and height of $40 a$ ) at random positions. This corresponds to a volume fraction of $\sim 1$ vol $\%$. Then, at $t^{*}=0$ the magnetic field is applied instantaneously. Soon after the application of the magnetic field short chains are formed that rapidly results in longer chains created by tip-to-tip aggregation. Five runs were performed with different initial configurations to obtain averages. In Fig. 11 we show the development of the magnetic energy $U^{*}$-normalized with the number of particles $N$-with time for five randomly generated structures. Very similar results are obtained independently of the initial configuration. Also, average energy values are shown for replicate simulations. As expected, the magnetic energy decreases toward a minimum energy value which is around $U^{*} / N=-2$. 


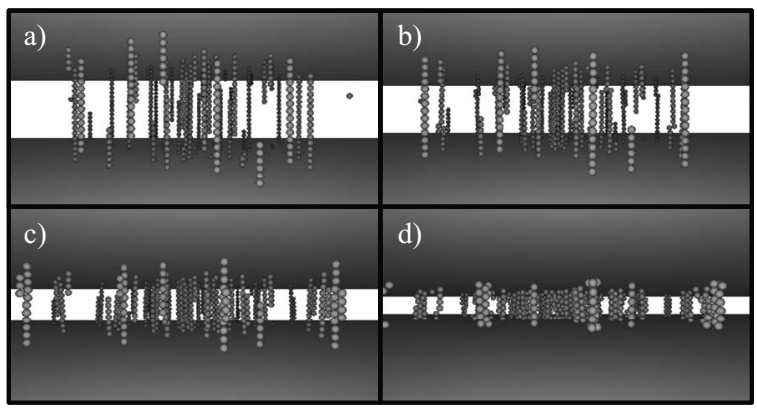

FIG. 12. Side view snapshots of $3 \mathrm{D}$ simulations squeezed at (a) $h=40 a$, (b) $h=32 a$, (c) $h=22 a$, and (d) $h=12 a ; \sim 1$ vol $\%, v^{*}=10^{-2}$.

Grown structures, previously annealed in the presence of a magnetic field, were later squeezed at a very slow constant speed of $v^{*}=10^{-2}$, resulting in a nonstationary flow where magnetic forces dominate over the hydrodynamic forces. To do so the gap was taken from an initial height of $40 a$ to a final height of $10 a$ according to Eq. (20). In Fig. 12 we show snapshots of the systems under slow compression at a constant approaching speed. As expected, the particles are able to find a minimum energy configuration in a more or less static condition. The straight chainlike structure is mostly conserved, and the excess stress is relieved through minor dislocations. Similar results were found in simulations by Lukkarinen and Kaski (1998).

In Fig. 13 the average magnetic energy is shown as a function of the compressive strain. At small strains, the energy increases slowly with the strain. However, violent fluctuations occur partially caused by macroscopic rearrangements of the chainlike structures in agreement with Chu et al. (2000) (see the inset in Fig. 13). At a certain strain value, the curve deviates from this behavior, with the energy increasing much more rapidly. By the derivation of magnetic energy [Eq. (23)], we calculated the normal force exerted by the field-induced structures. These results are shown in Fig. 9 for their com-

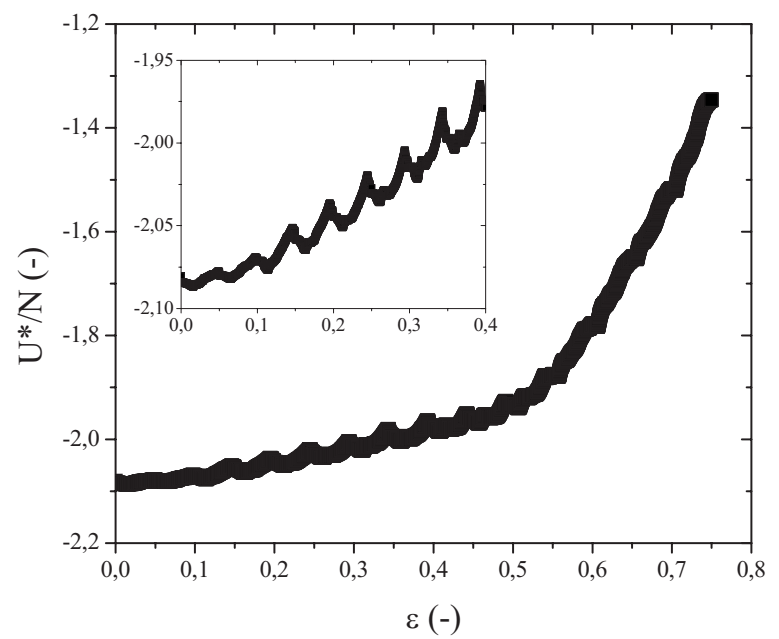

FIG. 13. Average magnetic energy normalized with the number of particles as function of the compressive strain. The periodic fluctuation of the normal force shown in the inset is associated to macroscopic rearrangements. 


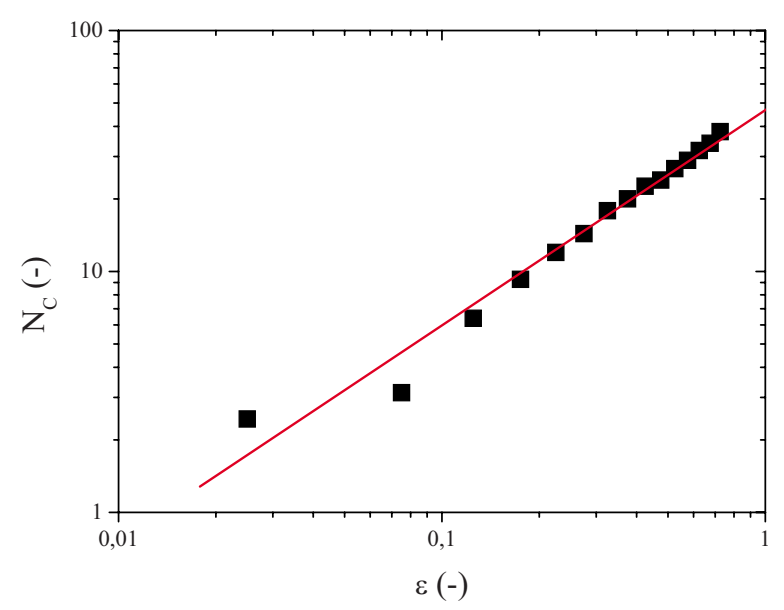

FIG. 14. Evolution of the number of percolating clusters, $N_{c}$, as a function of the compressive strain. The number of percolating clusters is strongly dependent on the connectivity criterion employed and roughly increases linearly with the strain. The red solid line is a linear fit with slope $0.89 \pm 0.04$ and correlation coefficient $r=0.99$.

parison with experimental and analytical model predictions. Interestingly, particle-level simulations qualitatively capture the normal force increase under compression. The extent of the quantitative agreement is somewhat surprising given the approximations in the simulation model.

To get a better understanding of the normal force evolution with the compressive strain, the kinetics of structure formation was first probed by following the number of percolating clusters, $N_{c}$, as a function of strain since the appearance of percolating clusters should be related to the appearance of normal forces. Cluster statistics were evaluated using the connectivity matrix method where sphere pairs with separations $r<2.2 a$ are considered directly connected. The number of percolating clusters, averaged over five simulation runs, is plotted as a function of compressive strain in Fig. 14. As observed, the number of percolating clusters is a linear function of the strain, and consequently it does not solely explain the significant increase in normal force for strains larger than $\varepsilon \sim 0.5$.

The number of percolating clusters is not the only contribution to the normal force since the compression-driven structural evolution of these percolating clusters does also contribute to the normal force. A simple estimation of the aspect ratio, thickness, and strength of the aggregates may be obtained through the $\Pi$ parameter. The existing correlation between this parameter and the resulting normal force is manifested by the linear relationship shown in Fig. 15. This suggests that the normal force increase under compression is governed by the average strength and thickness of the percolating aggregates. This was somehow expected because we calculated the normal force acting on the surfaces by taking the derivative of the magnetic energy.

\section{CONCLUSIONS}

It is well known that compression resistance of field-responsive fluids increases when the gap separation is reduced and compressive strain increases. Much of the work reported to date on the squeeze flow behavior of field-responsive fluids has focused on ER fluids under constant area and constant voltage operation. As a consequence, as the gap closes both the electric field intensity and particle concentration increase causing an 


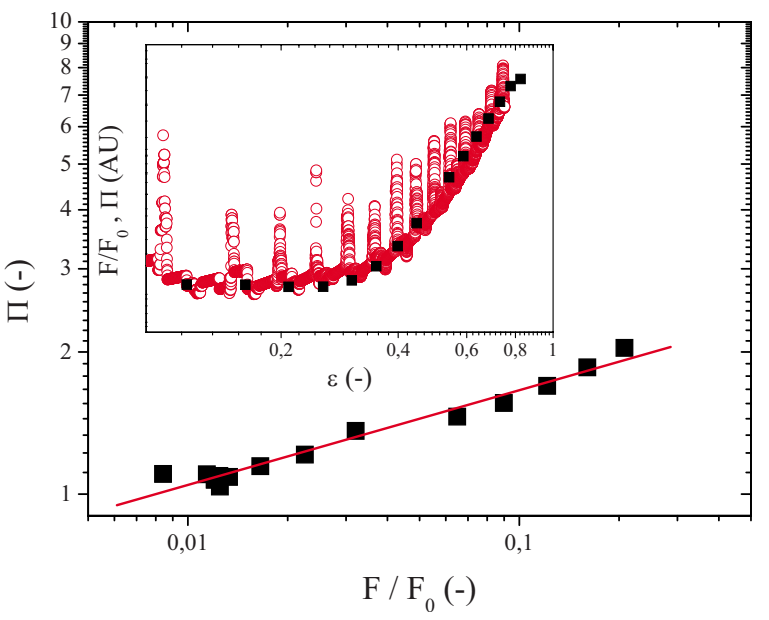

FIG. 15. $\Pi$ parameter as a function of the normalized normal force. The red solid line is a linear fit with slope $0.20 \pm 0.01$ and correlation coefficient $r=0.99$. Inset corresponds to the correlation between $F / F_{0}$ (black squares) and $\Pi$ (red open circles) versus the compressive strain dependence.

extra-hardening in the ER fluid. A significantly more simplified scenario results when compressing MR fluids under constant volume conditions. In this case, any compressionassisted hardening must be significantly dependent on the structural reorganization.

In this work the structural reorganization of MR fluids was proved using very small compressive speeds in the so-called filtration regime under the creeping flow approximation. Hence, the compressive stress can be looked as all contributed by the field-induced yield shear stress of the fluid, and the contribution of the viscous force to the compressive stress can be neglected. Even though it is well known that continuum Bingham and biviscous equations for ER fluids in oscillatory squeeze flow underestimate experimental results for the compressive stress under constant area operation, to date, this model had not been checked yet in the case of MR fluids. In this work we verified that Bingham and biviscous equations are able to reasonably predict compressive stresses in the case of MR fluids under constant volume operation. Furthermore, a standard micromechanical model consisting of a cubic network of single chains is able to predict the low-strain plateau as well by considering Hertzian contacts between the particles. However, the micromechanical model underestimates the normal force increase at large strains mainly due to the fact that only single chains are permitted in this model. This point was later demonstrated through the use of particle-level simulations. A close inspection of the experimental data reveals that macroscopic models slightly underestimate the compressive resistance when the gap separation decreases. This larger than expected increase of the normal force under compression can be explained in terms of a shear strengthening effect as proved here using combined squeeze and small-amplitude shear tests.

Experiments reported here concern low-concentration, no-slip, and very-slow-rate loading conditions where the strain rate although small is not constant during compression (maximum strain rate $\dot{\varepsilon} \sim 0.2 \mathrm{~s}^{-1}$ ). Simulation studies demonstrate that normal forces are strongly strain rate dependent. Actually, fast loading rates introduce an additional time scale which together with the structure evolution time scale makes the system very complicated. Commercial application of this technology requires the utilization of highly concentrated suspensions $(\sim 30$ vol \%), large loading rates, and partial slip con- 
ditions. So, in our opinion, future work should be devoted to better understand the effects of volume fraction, elongational rate, and slip at the surfaces in the squeeze flow behavior or MR fluids.

\section{ACKNOWLEDGMENTS}

This work was supported by MAT 2010-15101 project (Spain), by the European Regional Development Fund (ERDF), and by Junta de Andalucía Project No. P07-FQM02496 (Spain). J.P.S.-G. acknowledges financial support by the "Ministerio de Educación: Becas del Programa de Formación del Profesorado Universitario (FPU)" (Grant No. AP2008-02138). J.A.R-L. acknowledges support by the "Universidad de Granada. Becas de iniciación a la investigación" and "Ministerio de Educación. Becas de colaboración."

\section{References}

Andablo-Reyes, E., R. Hidalgo-Álvarez, and J. de Vicente, "A method for the estimation of the film thickness and plate tilt angle in thin film misaligned plate-plate rheometry," J. Non-Newtonian Fluid Mech. 165, 1419-1421 (2010).

Andablo-Reyes, E., R. Hidalgo-Álvarez, and J. de Vicente, "Controlling friction using magnetic nanofluids," Soft Matter 7, 880-883 (2011).

Bell, R. C., J. O. Karli, A. N. Vavreck, D. T. Zimmerman, G. T. Ngatu, and N. M. Wereley, "Magnetorheology of submicron diameter microwires dispersed in silicon oil," Smart Mater. Struct. 17, 015028 (2008).

Bird, R. B., Dynamics of Polymeric Liquids (Wiley, New York, 1987), 22.

Bossis, G., O. Volkova, S. Lacis, and A. Meunier, "Magnetorheology: Fluids, structures and rheology," in Ferrofluids: Magnetically Controllable Fluids and Their Applications, Lecture Notes in Physics Vol. 594, edited by S. Odenbach (Springer-Verlag, Berlin, 2002), 202-230.

Campanella, O. H., and M. Peleg, "Lubricated squeezing flow of a Newtonian liquid between elastic and rigid plates," Rheol. Acta 26(4), 396-400 (1987).

Chu, S.-H., S. J. Lee, and K. H. Ahn, "An experimental study on the squeezing flow of electrorheological suspensions," J. Rheol. 44(1), 105-120 (2000).

Collomb, J., F. Chaari, and M. Chaouche, "Squeeze flow of concentrated suspensions of spheres in Newtonian and shear-thinning fluids," J. Rheol. 48(2), 405-416 (2004).

Connelly, R. W., and J. Greener, "High-shear viscometry with a rotational parallel-disk device," J. Rheol. 29, 209-226 (1985).

Conrad, H., "Properties and design of electrorheological suspensions," MRS Bull. 23, 35-42 (1998).

Covey, G. H., and B. R. Stanmore, "Use of the parallel-plate plastometer for the characterization of viscous fluids with a yield stress," J. Non-Newtonian Fluid Mech. 8, 249-260 (1981).

de Gans, B. J., C. Blom, A. P. Philipse, and J. Mellema, "Linear viscoelasticity of an inverse ferrofluid," Phys. Rev. E 60, 4518-4527 (1999).

de Vicente, J., D. J. Klingenberg, and R. Hidalgo-Álvarez, "Magnetorheological fluids: A review," Soft Matter 7, 3701-3710 (2011).

de Vicente, J., M. T. López-López, J. D. G. Durán, and G. Bossis, “A slender-body micromechanical model for viscoelasticity of magnetic colloids: Comparison with preliminary experimental data," J. Colloid Interface Sci. 282, 193-201 (2005).

de Vicente, J., M. T. López-López, J. D. G. Durán, and F. González-Caballero, "Shear flow behavior of confined magnetorheological fluids at low magnetic field strengths," Rheol. Acta 44, 94-103 (2004).

de Vicente, J., M. T. López-López, F. González-Caballero, and J. D. G. Durán, "A rheological study of the stabilization of magnetizable colloidal suspensions by addition of silica nanoparticles," J. Rheol. 47(5), 1093-1109 (2003). 
de Vicente, J., and J. Ramírez, "Effect of friction between particles in the dynamic response of model magnetic structures,” J. Colloid Interface Sci. 316, 867-876 (2007).

de Vicente, J., J. P. Segovia-Gutiérrez, E. Andablo-Reyes, F. Vereda, and R. Hidalgo-Álvarez, "Dynamic rheology of sphere- and rod-based magnetorheological fluids," J. Chem. Phys. 131, 194902 (2009).

de Vicente, J., F. Vereda, J. P. Segovia-Gutiérrez, M. P. Morales, and R. Hidalgo-Álvarez, "Effect of particle shape in magnetorheology,” J. Rheol. 54, 1337-1343 (2010).

Dienes, G. J., and H. F. Klemm, "Theory and application of the parallel plate plastometer," J. Appl. Phys. 17, 458-471 (1946).

Ekwebelam, C. C., and H. See, "Using oscillatory shear to probe the effects of bidispersity in inverse ferrofluids," Korea-Aust. Rheol. J. 19, 35-42 (2007).

El Wahed, A. K., J. L. Sproston, and R. Stanway, "The performance of an electrorheological fluid in dynamic squeeze flow under constant voltage and constant field," J. Phys. D: Appl. Phys. 31, 2964-2974 (1998).

El Wahed, A. K., J. L. Sproston, and R. Stanway, "The performance of an electrorheological fluid in dynamic squeeze flow: The influence of solid phase size," J. Colloid Interface Sci. 211, 264-280 (1999).

El Wahed, A. K., J. L. Sproston, R. Stanway, and E. W. Williams, "An improved model of ER fluids in squeeze-flow through model updating of the estimated yield stress," J. Sound Vib. 268, 581-599 (2003).

El Wahed, A. K., J. L. Sproston, and E. W. Williams, "The effect of a time-dependent electric field on the dynamic performance of an electrorheological fluid in squeeze," J. Phys. D: Appl. Phys. 33, 2995-3003 (2000).

Engmann, J., C. Servais, and A. S. Burbidge, "Squeeze flow theory and applications to rheometry: A review," J. Non-Newtonian Fluid Mech. 132, 1-27 (2005).

Gartling, D. K., and N. Phan-Thien, "A numerical simulation of a plastic fluid in a parallel-plate plastometer," J. Non-Newtonian Fluid Mech. 14, 347-360 (1984).

Ginder, J. M., "Behavior of magnetorheological fluids," MRS Bull. 23(8), 26-29 (1998).

Gong, H., and M. K. Lim, "Experimental investigation on tension and compression properties of an electrorheological material,” J. Intell. Mater. Syst. Struct. 7, 89-96 (1996).

Havelka, K. O., and J. W. Pialet, "Electrorheological technology: The future is now," CHEMTECH 26, 36-45 (1996).

Heine, M. C., J. de Vicente, and D. J. Klingenberg, "Thermal transport in sheared electro- and magnetorheological fluids," Phys. Fluids 18, 023301 (2006).

Jiles, D., Introduction to Magnetism and Magnetic Materials (Chapman and Hall, London, 1991).

Jolly, M. R., and J. D. Carlson, in Actuator 96, 5th International Conference on New Actuators, edited by H. Borgmann and K. Lenz (Axon Technologies Consult Gmb, Bremen, 1996).

Kim, D. H., S.-H. Chu, K. H. Ahn, and S. J. Lee, "Dynamic simulation of squeezing flow of ER fluids using parallel processing,” Korea-Aust. Rheol. J. 11(3), 233-240 (1999).

Kim, S. G., J. Y. Lim, J. H. Sung, H. J. Choi, and Y. Seo, "Emulsion polymerized polyaniline synthesized with dodecylbenzenesulfonic acid and its electrorheological characteristics: Temperature effect," Polymer 48, 6622-6631 (2007).

Klingenberg, D. J., F. van Swol, and C. F. Zukoski, "Dynamic simulation of electrorheological suspensions," J. Chem. Phys. 91, 7888-7895 (1989).

Klingenberg, D. J., F. van Swol, and C. F. Zukoski, "The small shear rate response of electrorheological suspensions. I. Simulation in the point-dipole limit," J. Chem. Phys. 94, 6160-6169 (1991).

Kulkarni, P., C. Ciocanel, S. L. Vieira, and N. Naganathan, "Study of the behavior of MR fluids in squeeze, torsional and valve modes," J. Intell. Mater. Syst. Struct. 14, 99-104 (2003).

Laeuger, J., K. Wollny, H. Stettin, and S. Huck, "A new device for the full rheological characterization of magneto-rheological fluids,” Int. J. Mod. Phys. B 19, 1353-1359 (2005).

Laun, H. M., M. Rady, and O. Hassager, "Analytical solutions for squeeze flow with partial wall slip," J. Non-Newtonian Fluid Mech. 81, 1-15 (1999).

Laun, H. M., G. Schmidt, and C. Gabriel, "Reliable plate-plate MRF magnetorheometry based on validated radial magnetic flux density profile simulations," Rheol. Acta 47, 1049-1059 (2008).

Lee, C. Y., and C. Y. Wen, "The oscillatory squeeze flow of electrorheological fluid considering the inertia effect," Smart Mater. Struct. 11, 553-560 (2002). 
Lipscomb, G. G., and M. M. Denn, "Flow of Bingham fluids in complex geometries," J. Non-Newtonian Fluid Mech. 14, 337-346 (1984).

Lukkarinen, A., and K. Kaski, "Computational studies of compressed and sheared electrorheological fluid," J. Phys. D: Appl. Phys. 29, 2729-2732 (1996).

Lukkarinen, A., and K. Kaski, "Simulation studies of electrorheological fluids under shear, compression, and elongation loading,” J. Appl. Phys. 83(3), 1717-1725 (1998).

Lynch, R., Y. Meng, and F. E. Filisko, "Compression of dispersions to high stress under electric fields: Effects of concentration and dispersing oil," J. Colloid Interface Sci. 297, 322-328 (2006).

Mazlan, S. A., K. H. Ekreem, and A. G. Olabi, "An investigation of the behaviour of magnetorheological fluids in compression mode,” J. Mater. Process. Technol. 201, 780-785 (2008).

McIntyre, E. C., "Compression of smart materials: Squeeze flow on electrorheological and magnetorheological fluids," Ph.D. thesis, The University of Michigan, 2008.

McIntyre, E. C., and F. E. Filisko, "Squeeze flow of electrorheological fluids under constant volume," J. Intell. Mater. Syst. Struct. 18, 1217-1220 (2007).

McIntyre, E. C., and F. E. Filisko, "Filtration in electrorheological suspensions related to the Peclet number," J. Rheol. 54(3), 591-603 (2010).

Meng, Y., and F. E. Filisko, "Unidirectional compression of electrorheological fluids in electric fields," J. Appl. Phys. 98, 074901 (2005).

Monkman, G. J., "Design and application of magneto-rheological fluid,” J. Phys. D: Appl. Phys. 28, 588-593 (1995).

Olabi, A. G., and A. Grunwald, "Design and application of magneto-rheological fluid," Mater. Des. 28, 26582664 (2007).

Park, B. J., F. F. Fang, and H. J. Choi, "Magnetorheology: Materials and application," Soft Matter 6, 52465253 (2010).

Park, B. J., I. B. Jang, H. J. Choi, A. Pich, S. Bhattacharya, and H.-J. Adler, "Magnetorheological characteristics of nanoparticle-added carbonyl iron system,” J. Magn. Magn. Mater. 303, e290-e293 (2006).

Ramos, J., J. de Vicente, and R. Hidalgo-Álvarez, "Small-amplitude oscillatory shear magnetorheology of inverse ferrofluids," Langmuir 26(12), 9334-9341 (2010).

Ramos, J., D. J. Klingenberg, R. Hidalgo-Álvarez, and J. de Vicente, "Steady shear magnetorheology of inverse ferrofluids," J. Rheol. 55, 127-152 (2011).

Rankin, P. J., J. M. Ginder, and D. J. Klingenberg, "Electro- and magnetorheology," Curr. Opin. Colloid Interface Sci. 3, 373-381 (1998).

Raphaelides, S. N., and A. Gioldasi, "Elongational flow studies of set yogurt," J. Food. Eng. 70(4), 538-545 (2004).

Saldivar-Guerrero, R., R. Richter, I. Rehberg, N. Aksel, L. Heymann, and O. S. Rodríguez-Fernández, "Viscoelasticity of mono- and polydisperse inverse ferrofluids," J. Chem. Phys. 125, 084907 (2006).

Scott, J. R., Trans. Inst. Rubber Ind., 4, 347 (1929).

See, H., "Field dependence of the response of a magnetorheological suspension under steady shear flow and squeezing flow," Rheol. Acta 42, 86-92 (2003).

See, H., J. S. Field, and B. Pfister, "The response of electrorheological fluid under oscillatory squeeze flow," J. Non-Newtonian Fluid Mech. 84, 149-158 (1999).

See, H., S. Mackenzie, and B. T. Chua, "Effect of compression on the response of a magneto-rheological suspension," Korea-Aust. Rheol. J. 18(3), 121-126 (2006).

Sproston, J. L., S. G. Rigby, E. W. Williams, and R. Stanway, "A numerical simulation of electrorheological fluids in oscillatory compressive squeeze-flow,” J. Phys. D: Appl. Phys. 27, 338-343 (1994b).

Sproston, J. L., R. Stanway, E. W. Williams, and S. G. Rigby, "The electrorheological automotive engine mount," J. Electrost. 32, 253-259 (1994a).

Stanway, R., J. L. Sproston, M. J. Prendergast, J. R. Case, and C. E. Wilne, "ER fluids in the squeeze-flow mode: An application to vibration isolation,” J. Electrost. 28, 89-94 (1992).

Stanway, R., J. L. Sproston, and N. G. Stevens, "Non-linear modelling of an electro-rheological vibration damper," J. Electrost. 20, 167-184 (1987).

Stefan, J., "Versuche uber der scheinbare adhasion," Sitz. Kais. Akad. Wiss Math. Nat. Wien 69(2), 713-735 
(1874).

Tang, X., X. Zhang, R. Tao, and Y. Rong, "Structure-enhanced yield stress of magnetorheological fluids," J. Appl. Phys. 87(5), 2634-2638 (2000).

Tao, R., Y. C. Lan, and X. Xu, "Structure-enhanced yield shear stress in electrorheological fluids," Int. J. Mod. Phys. B 16, 2622-2628 (2002).

Tian, Y., Y. Meng, H. Mao, and S. Wen, "Electrorheological fluid under elongation, compression, and shearing," Phys. Rev. E 65, 031507 (2002a)

Tian, Y., Y. Meng, H. Mao, and S. Wen, "Mechanical property of electrorheological fluid under step compression,” J. Appl. Phys. 92, 6875-6879 (2002b).

Tian, Y., S. Wen, and Y. Meng, "Compressions of electrorheological fluids under different initial gap distances," Phys. Rev. E 67, 051501 (2003).

Tian, Y., M. Zhang, X. Zhu, Y. Meng, and S. Wen, "Ultrahigh yield stress in a general electrorheological fluid under compression," Smart Mater. Struct. 19, 035009 (2010).

Tian, Y., and Q. Zou, "Normalized method for comparing tensile behaviors of electrorheological fluids," Appl. Phys. Lett. 82(26), 4836-4838 (2003).

Vereda, F., J. de Vicente, and R. Hidalgo-Álvarez, "Influence of a magnetic field on the formation of magnetite particles via two precipitation methods," Langmuir 23, 3581-3589 (2007).

Vereda, F., J. de Vicente, and R. Hidalgo-Álvarez, "Physical properties of elongated magnetic particles: Magnetization and friction coefficient anisotropies," ChemPhysChem 10, 1165-1179 (2009).

Vieira, S. L., M. Nakano, R. Oke, and T. Nagata, “Tension and compression of electrorheological fluid,” Int. J. Mod. Phys. B 15, 714-722 (2001).

Volkova, O., G. Bossis, M. Guyot, V. Bashtovoi, and A. Reks, "Magnetorheology of magnetic holes compared to magnetic particles," J. Rheol. 44, 91-104 (2000).

Wang, X., and F. Gordaninejad, "Study of magnetorheological fluids at high shear rates," Rheol. Acta 45, 899-908 (2006).

Williams, E. W., S. G. Rigby, J. L. Sproston, and R. Stanway, "Electrorheological fluids applied to an automotive engine mount,” J. Non-Newtonian Fluid Mech. 47, 221-238 (1993).

Wollny, K., J. Lauger, and S. Huck, "Magneto sweep-A method for characterizing the viscoelastic properties of magneto-rheological fluids," Appl. Rheol. 12, 25-31 (2002).

Wu, C. W., and H. Conrad, "Shear strength of electrorheological particle clusters," Mater. Sci. Eng., A 248, 161-164 (1998).

Yang, F., "Tension and compression of electrorheological fluid,” J. Colloid Interface Sci. 192, 162-165 (1997).

Zhang, M. L., Y. Tian, J. L. Jiang, X. L. Zhu, Y. G. Meng, and S. Z. Wen, "Compression enhanced shear yield stress of electrorheological fluid," Chin. Phys. Lett. 26(4), 048301 (2009).

Zhang, X. Z., X. L. Gong, P. Q. Zhang, and Q. M. Wang, "Study on the mechanism of the squeeze-strengthen effect in magnetorheological fluids," J. Appl. Phys. 96(4), 2359-2364 (2004). 